\title{
Recognition Method Based on Wigner-Hough Transform for Poly-Phase Code Radar Signal
}

\author{
Wenxu Zhang, Bing Wang, Fuli Sun \\ College of Information and Communication Engineering, Harbin Engineering University, Harbin, China \\ Email: zhangwenxu@hrbeu.edu.cn,wangbing2016@hrbeu.edu.cn, s316087018@hrbeu.edu.c
}

How to cite this paper: Zhang, W.X., Wang, B. and Sun, F.L. (2017) Recognition Method Based on Wigner-Hough Transform for Poly-Phase Code Radar Signal. Int. J. Communications, Network and System Sciences, 10, 128-137.

https://doi.org/10.4236/ijcns.2017.108B014

Received: May 24, 2017

Accepted: August 11, 2017

Published: August 14, 2017

\begin{abstract}
In order to solve the recognition of polyphase code radar signal, this paper gives two methods based on Frank code, i.e. the high-order spectrum recognition method and the fractional Fourier transform (FRFT) method, by analyzing the micro characteristics of polyphase code signals in time and frequency domain respectively. And a recognition algorithm based on Wigner-Hough transform (WHT) is developed in this paper. We verify the validity of each method by computer simulation and give relative merits and demerits. A set of results demonstrate that the algorithm based on Wigner-Hough transform has better recognition performance in low signal-to-noise (SNR) than others.
\end{abstract}

\section{Keywords}

Radar Signal Processing, Polyphase Code, Fractional Fourier Transform, Wigner-Hough Transform

\section{Introduction}

In recent years, a class of polyphase code radar signals (Frank, P1, P2, P3, P4 code) which is derived from linear frequency modulation (LFM) have wide applications in field of radar anti-interference system. Combining the doppler tolerance features of LFM and high range resolution of phase encoding, polyphase code signals have excellent pulse compression performance [1]. While in electronic battlefield, signals are intertwined and highly dense and there is a variety of modulation methods, which causes no priori in detection signals [2]. Thus, it is significant to analyze the characteristics of polyphase code signals from complicated signals and develop an efficient and feasible method for signal detection and recognition.

Polyphase code signals have similar time-frequency characteristics with LFM signals. Referring to the methods of LFM recognition, a mass of research results 
have been acquired for polyphase code signals detection and parameter estimation. For example, paper [3] proposed a way to detect the LFM polyphase code signals by combining Wigner-Ville distribution with Hough transform. It was verified by simulation and the results showed that this method had preferable anti-noise performance. In order to detect the polyphase code signals and estimate parameters accurately in low signal-to-noise ratio (SNR), paper [4] developed a method based on WHT. It was achieved by improving the kernel function of WHT and accumulating energy of multiple ridge lines of WVD in polyphase code signals. The experimental results demonstrated that this approach proposed was effective to detect and estimate the polyphase code signals in low SNR and it was not affected by encoding types. And a signal processing method based on integral quadratic phase function and fractional Fourier transform was proposed by DuQin [5]. It had a clear advantage of small calculation and little cross interference items. So the accuracy of parameter estimation was enhanced obviously. A kind of detection to multiphase coded pulse compression signal using Radon-Ambiguity transform (RAT) was given in paper [6], but there were no methods to estimate waveform parameters.

In this paper, we focus on the detection and estimation of Frank code in polyphase code signals by high-order spectrum recognition method, fractional Fourier transform (FRFT) and Wigner-Hough transform (WHT) respectively. The detection based on high-order spectrum recognition method is easy to practice theoretically but difficult implementation on hardware design. The one based on FRFT is achieved by rotating coordinate axis to get the optimum status of signal gathering. It has an excellent filter performance. The detection and parameter estimation to polyphase code signals using WHT are done by taking example by detecting LFM signal using WHT. The recognition performance is great especially in low SNR.

\section{Analysis of Polyphase Code Signal}

Phase coded signal is a kind of pulse radar signal, usually expressed in the form of complex signal

$$
S(t)=A \exp \left[\mathrm{j}\left(2 \pi f_{0} t+\varphi_{p, q}\right)\right]
$$

where $A$ is the signal amplitude. $f_{0}$ is the carrier frequency of signal. $\varphi_{p, q}$ is a phase code sequence changing with time, which is related to the type of encoding modulation used.

Supposing a coding cycle is $T$, which is divided into $N_{c}$ equal width subpulses, and $N_{c}=M^{2} . T_{c}$ represents the width of the code. The coding period is $T=N_{c} T_{c}$. Table 1 shows the modulation phase of several common polyphase code pulse signals.

The paper mainly uses Frank code as an example to carry on the simulation and the discussion.

The phase transition diagram for the Frank code when $N_{c}=64$ was shown in (a) of Figure 1; (b) is the time domain waveform of Frank code; (c) is the spectrum of Frank code. 
Table 1. Modulation phase of poly-phase code.

\begin{tabular}{|c|c|}
\hline Frank & $\varphi_{p, q}=\frac{2 \pi}{M}(p-1)(q-1) ; q=1,2, \ldots, M, p=1,2, \ldots, M$ \\
\hline P1 & $\varphi_{p, q}=-\frac{\pi}{M}[M+1-2 q][M(q-1)+(p-1)] ; q=1,2, \ldots, M, p=1,2, \ldots, M$ \\
\hline P2 & $\varphi_{p, q}=\frac{\pi}{2 M}[M+1-2 q][m+1-2 p] ; q=1,2, \ldots, M, p=1,2, \ldots, M(M$ is an even number $)$ \\
\hline P3 & $\varphi_{p, q}=\frac{\pi}{N_{c}}(q-1)^{2} ; q=1,2, \ldots, N_{c}$ \\
\hline P4 & $\varphi_{p, q}=\frac{\pi}{N_{c}}(q-1)\left(q-1-N_{c}\right) ; q=1,2, \ldots, N_{c}$ \\
\hline
\end{tabular}

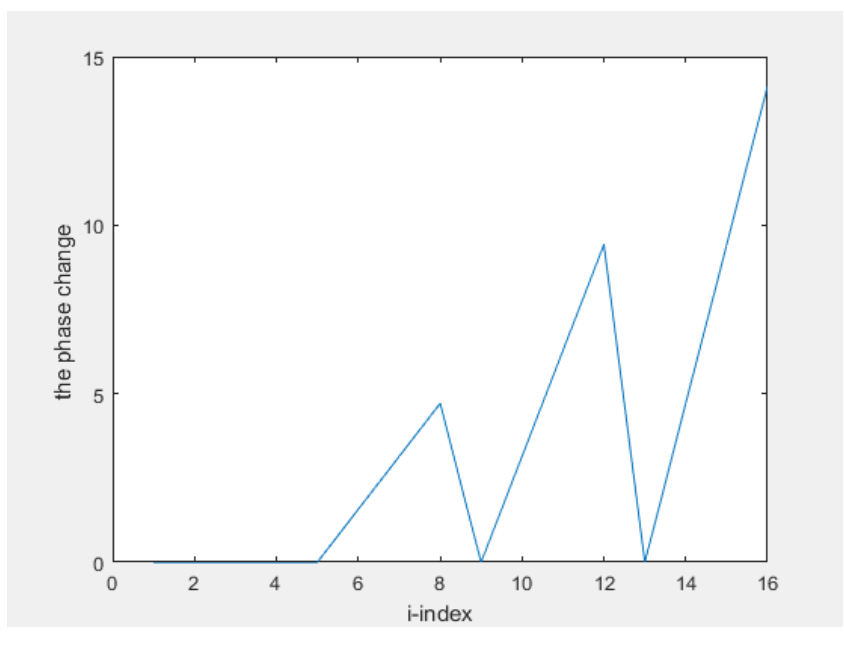

(a)

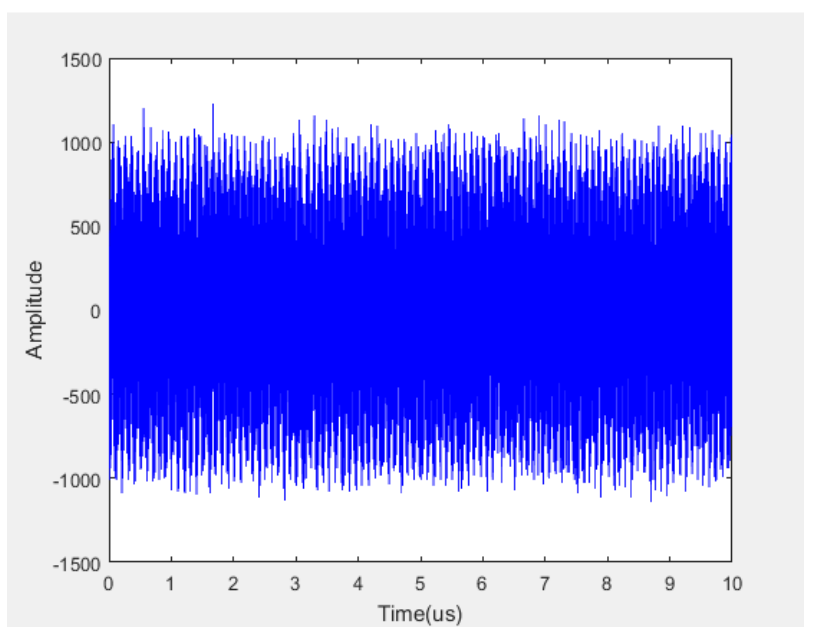

(b)

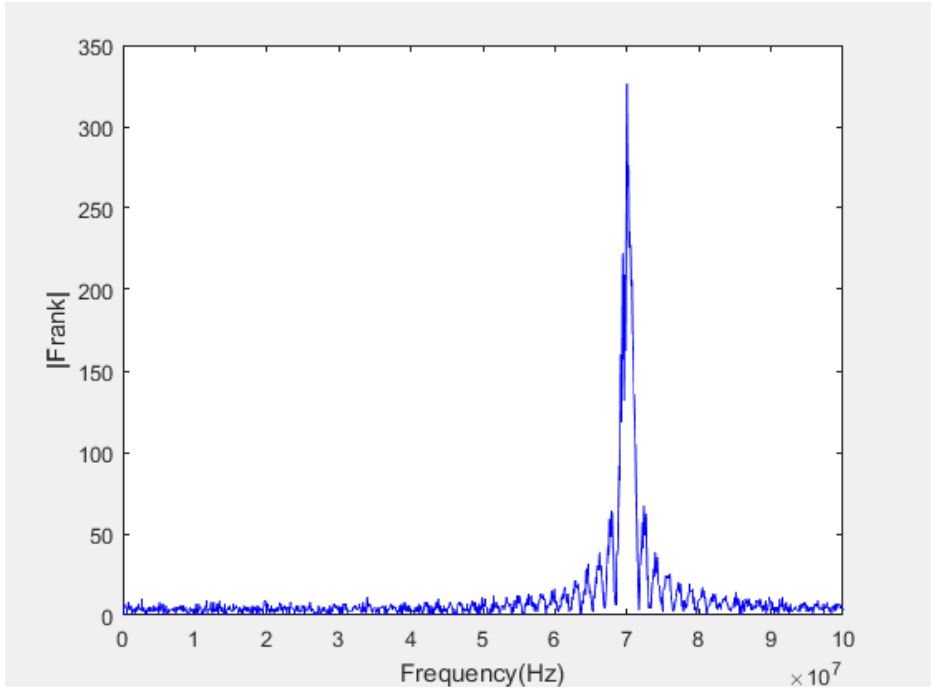

(c)

Figure 1. State Distribution of Frank code. (a) Phase-changing of Frank Code. (b) Time Domain Waveform. (c) Spectrum Distribution. 


\section{Basic Recognition Method of Polyphase Code Signal}

The high-order spectrum recognition method and the FRFT recognition method are widely used in LFM and two-phase and four-phase coded signals, and they have been gradually applied to the polyphase code signal. The paper introduces the application of the two methods in the polyphase code firstly.

\subsection{High-Order Spectrum Recognition Method}

Perform a quadratic operation on Frank codes

$$
[s(t)]^{2}=A^{2} \cdot \exp \left[\mathrm{j}\left(2 \pi \cdot 2 f_{0} t+2 \varphi_{p, q}\right)\right]
$$

Remove the mean after the quadratic operation, and then take the Fourier transform of operation results. From the following simulation, Figure 3, it can be seen that the side lobes are well reduced, and making it easier to find the main peak at the time of detection. Perform the fourth-order calculation for Frank code, it can be seen the inhibitory effect for side-lobes is better than the secondorder. However, it is difficult to accurately determine the arrival of the signal in low signal to noise ratio, the general use of correlation detection. Perform quadratic or quadratic operation for the received signal by the above method, then do Fourier transform operation, theoretically it can achieve a better detection results [7].

But, the second-order and the fourth-order computing are not easy to achieve in the hardware design. The consumption of hardware resources of the detection system is very high when do the corresponding calculation and recording of multiphase code signals with certain pulse width. And the output of the Fourier transform of the Frank code doesn't make sense for subsequent data analysis.

\subsection{Recognition Method Based on Fractional Fourier Transform}

The Fractional Fourier Transform (FRFT) of the signal $s(t)$ is defined as

$$
S_{p}(u)=F^{p}[s(t)]=\int_{-\infty}^{\infty} s(t) K_{p}(t, u) \mathrm{d} t
$$

where $p$ represents the FRFT order, $F^{p}[]$ represents the FRFT operator, $K_{\alpha}(t, u)$ represents the FRFT transform the kernel.

$$
\begin{gathered}
K_{p}(t, u)=\left\{\begin{array}{l}
A_{\alpha} \exp \left[\mathrm{j} \pi\left(u^{2} \cot \alpha-2 u t \csc \alpha+t^{2} \cot \alpha\right)\right] \alpha \neq n \alpha \\
\delta(t-u) \alpha=2 n \pi \\
\delta(t+u) \alpha=(2 n \pm 1)
\end{array}\right. \\
A_{\alpha}=\frac{\exp [-\mathrm{j} \pi \operatorname{sgn}(\sin \alpha) / 4+\mathrm{j} \alpha / 2]}{|\sin \alpha|^{1 / 2}}
\end{gathered}
$$

The rotation angle is $\alpha=p \pi / 2$.

The FRRT of the signal can be seen as the rotation of the axis of the signal on the time-frequency plane around the origin by counterclockwise rotation of the $\alpha$-angle to the $u$-axis. Different rotation angles correspond to different frequency modulation bases. And the optimum aggregation characteristics can be achieved at the optimum rotation angle. Figure 2 shows the degree of ag- 


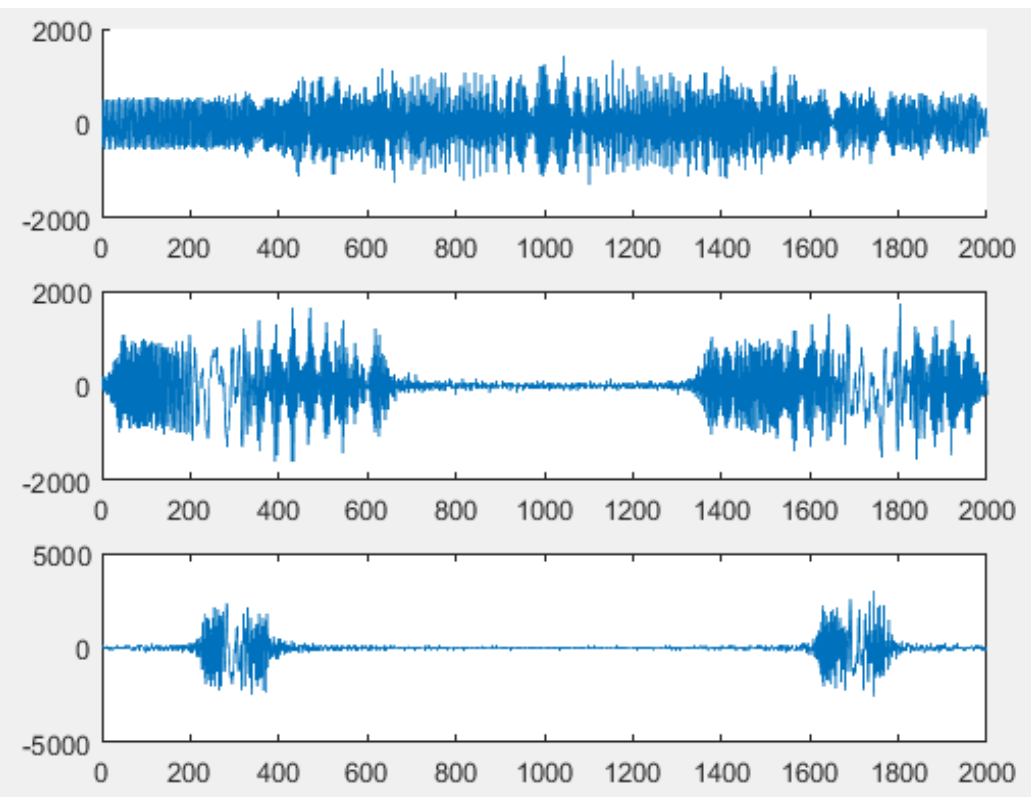

Figure 2. Aggregation degree of signals under different orders.

gregation of signals at different orders [8].

The FRFT three-dimensional distribution of the Frank code signal is shown as Figure 4. On the parameter plane $(\alpha, u)$, the Frank code signals have two high spikes with the same $\alpha$ coordinates. Let the coordinates be $\alpha_{0}$, which is the best fractional rotation angle of Frank code. The distances of adjacent peaks are both $\Delta u$. Combining the time-frequency distribution of Frank code and the FRFT domain spectrum under angle $\alpha_{0}$, it can be given as follows.

The bandwidth of the Frank code signal

$$
B=\frac{\Delta u}{\sin \alpha_{0}}
$$

The coding cycles of codes

$$
T=\frac{\Delta u}{\cos \alpha_{0}}
$$

The width of codes

$$
\tau_{c}=\frac{1}{B}
$$

The number of codes

$$
N_{c}=\frac{T}{\tau_{c}}
$$

The estimated value of carrier frequency

$$
\hat{f}_{c}=\left(\sum_{k=1}^{k} \hat{f}_{0 k}\right) / k
$$

where $f_{0 k}$ is the frequency value at the intersection of the main ridge line and the frequency axis of the $k$ bar of the Frank code signal, and the estimate is 
$\hat{f}_{0 k}=\hat{u}_{0 k} \csc \hat{\alpha}_{0} .\left\{\hat{\alpha}_{0}, \hat{u}_{0 k}\right\}$ is the spike coordinate of the signal on the parameter plane. $k=1,2, \cdots, N, N$ is the number of signal spike.

The time-frequency curve of the Frank code signal can be regarded as the time-frequency curve of the LFM signal shifted by a certain delay. So we can draw on the LFM signal detection method based on fractional Fourier transform [9].

\section{Method of Polyphase Code Recognition Based on Wigner-Hough}

Wigner distribution is a time-frequency analysis method, which is usually used to analyze the non-stationary signal. The Hough transform is a method for detecting the graphical features in image processing. The Frank code is detected by performing the Hough transform for the Wigner distribution results, and meanwhile the parameters of the signal can be estimated [10].

The Hough transforms' principle of extracting straight lines.

On any point in the image space $\left(x_{0}, y_{0}\right)$, using the transformation

$$
\rho=x \cos \theta+y \sin \theta
$$

to transform it into a curve, $\rho=x_{0} \cos \theta+y_{0} \sin \theta$, in the parameter space $(\rho, 0)$. The $n$ points in the same line by the transformation can get the corresponding $n$ bar curve in the parameter space.

This $n$ curves go through the same point $\left(\rho_{0}, \theta_{0}\right)$. Finding this point in the parameter space it means to have found the corresponding line in the image space.

The time-frequency distribution of the detected signal is taken as an image, then using Hough transform to detect straight line in this image. This translates the problem of the detection and parameter estimation of the Frank code signal into the problem of searching for the local maximum and its corresponding coordinate in the parameter space. The time-frequency distribution of the Frank code signal is a number of parallel ridge lines. And it exhibited multiple peak points on the parameter plane after Hough transform [11].

The definition of signal's WVD

$$
W_{x}(t, f)=\int_{-\infty}^{\infty} x(t+\tau / 2) x^{*}(t-\tau / 2) e^{-j 2 \pi f \tau} \mathrm{d} \tau
$$

Perform Wigner-Hough transform of the signal by a straight line equation, $f^{\prime}=f+g t$.

$$
\begin{aligned}
\operatorname{WHT}_{x}(f, g) & =\int_{-\infty}^{\infty} \int_{-\infty}^{\infty} W_{x}\left(t, f^{\prime}\right) \delta\left(f^{\prime}-f-g t\right) \mathrm{d} t \mathrm{~d} f \\
& =\int_{-\infty}^{\infty} W_{x}(t, f+g t) \mathrm{d} t \\
& =\int_{-\infty}^{\infty} \int_{-\infty}^{\infty} x(t+\tau / 2) x^{*}(t-\tau / 2) e^{-j 2 \pi(f+g t) \tau} \mathrm{d} t \mathrm{~d} \tau
\end{aligned}
$$

\section{Computer Simulation and Performance Analysis}

In order to verify the effectiveness and feasibility of the method, using Frank code to do the following simulation analysis. The center frequency is $f_{i}=70 \mathrm{MHz}$, 
The carrier frequency is $f_{s}=200 \mathrm{MHz}$.

The two spectrum distribution figures can be got by performing the secondorder and the fourth-order operation for Frank code and then doing Fourier transform after removing the mean value. They are shown as Figure 3. It can be seen the noise and side lobes are well suppressed through the method from the figure. Thus it verified the validity of the method based on high-order spectrum.

The FRFT three-dimensional distribution of the Frank code signal is shown as (a) of Figure 4, and its spectrum in the FRFT domain is shown as (b).

Due to phase quantization, the WVD distribution of Frank code is multi-ridge distribution on the Wigner time-frequency plane. The three-dimensional distribution of the signal through Wigner-Hough transform is shown as Figure 5. There are multiple spikes in the figure due to phase quantization.

When the noise is increased to $-2 \mathrm{~dB}$, the spectrum of the signal after each method is shown as follows.

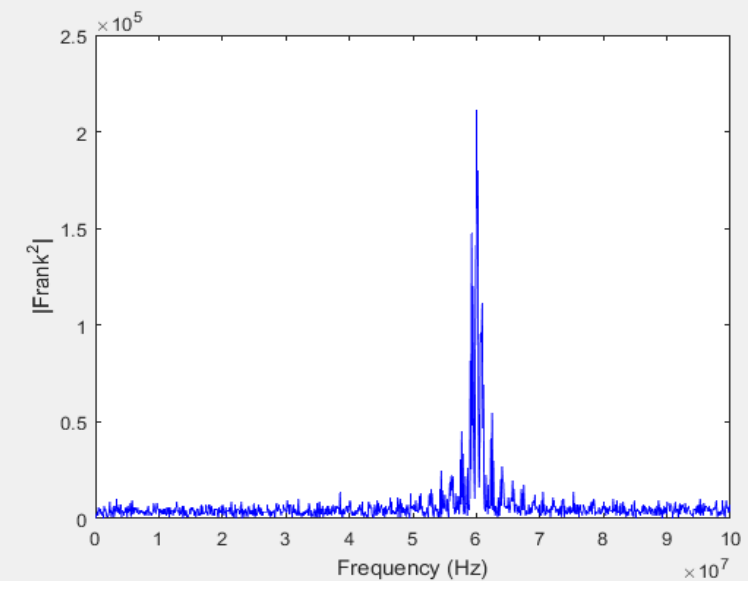

(a)

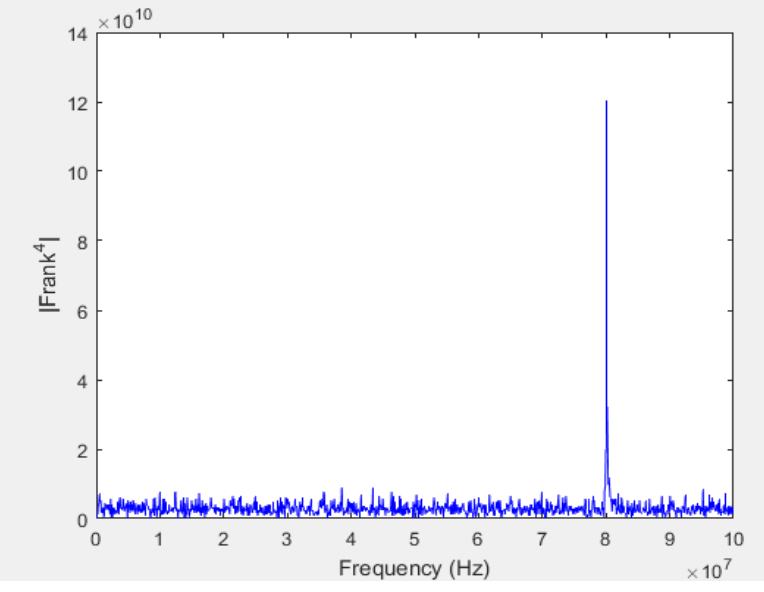

(b)

Figure 3. High-order spectrum distribution of frank code. (a) Second-order spectrum distribution. (b) Fourth-order spectrum distribution.

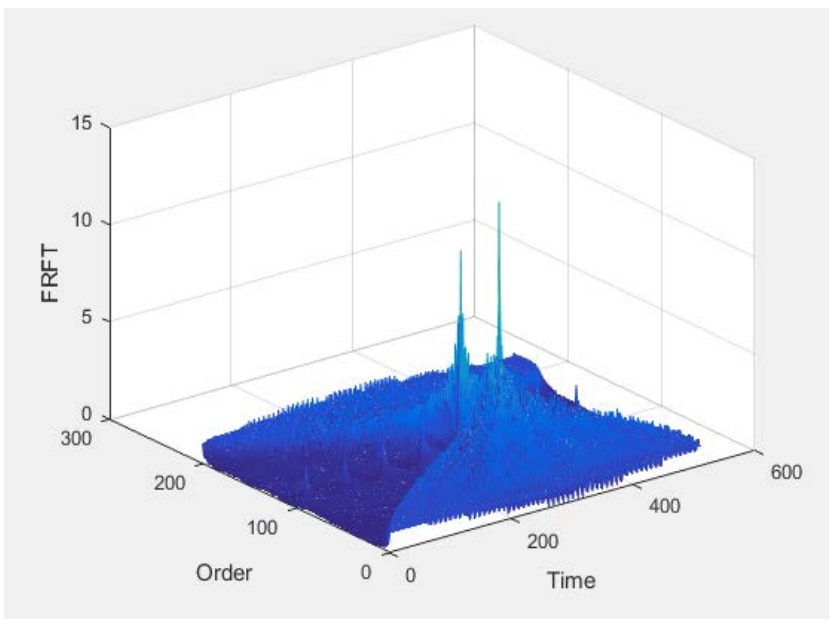

(a)

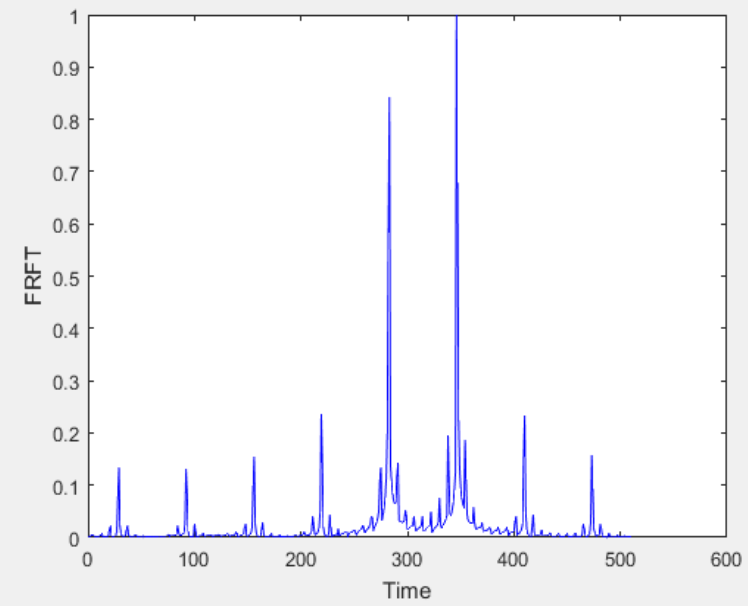

(b)

Figure 4. Spectrum distribution based on FRFT. (a) Fractional-order spectral distribution. (b) FRFT domain spectrum. 


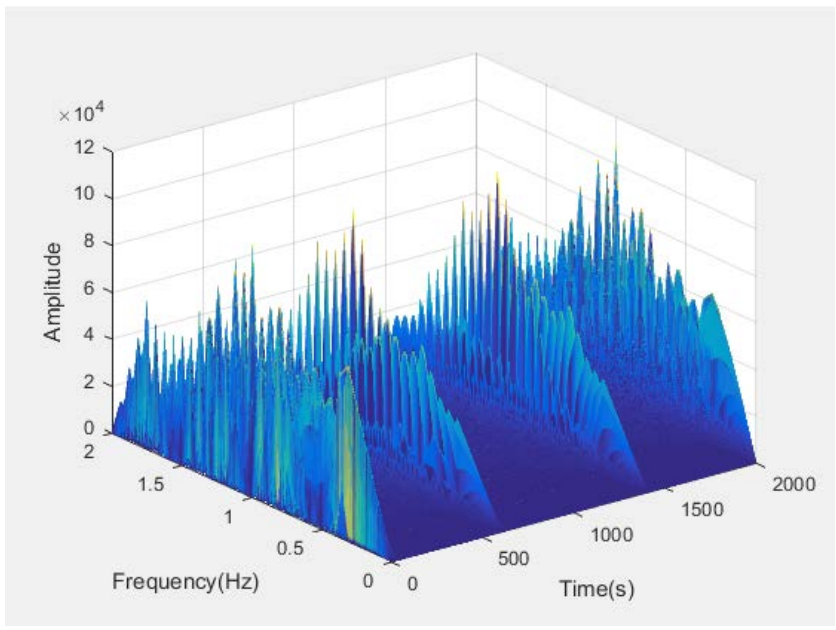

(a)

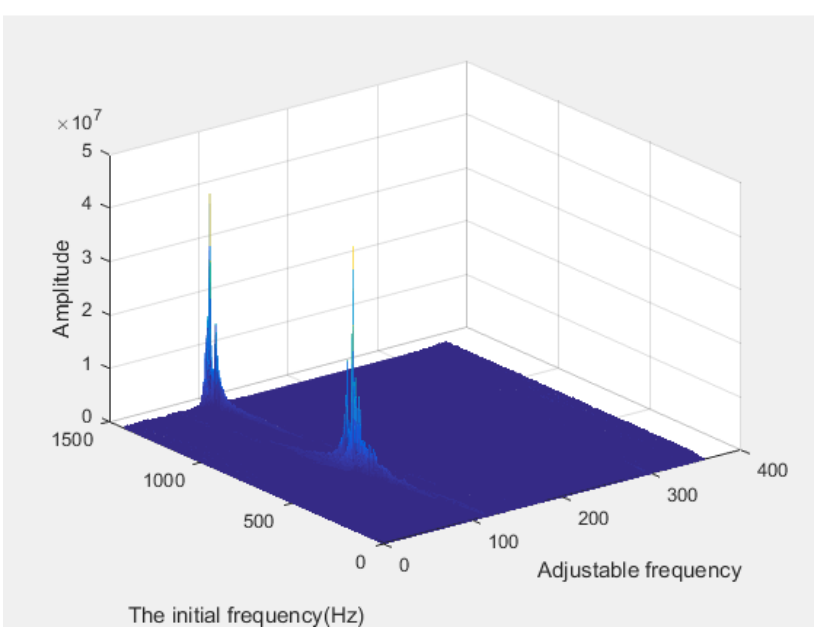

(b)

Figure 5. Wigner-ville distribution and wigner-hough distribution of frank code. (a) WVD. (b) WHT.

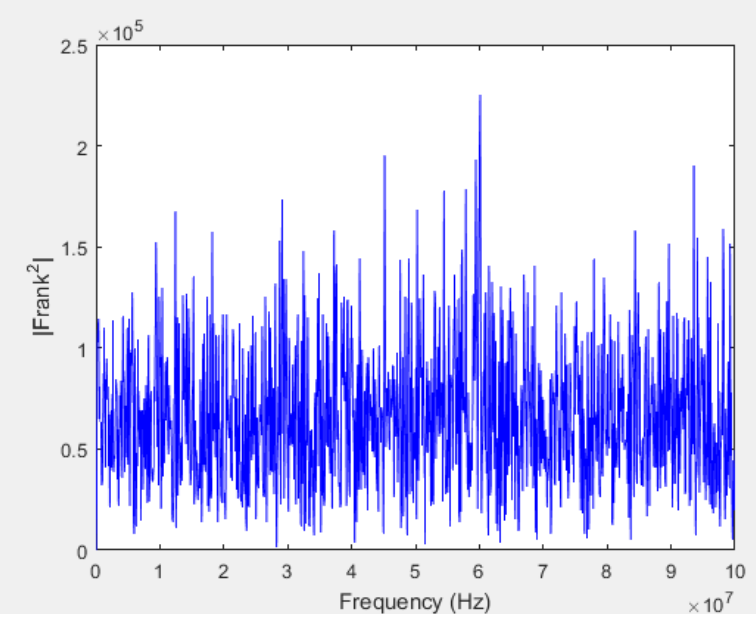

(a)

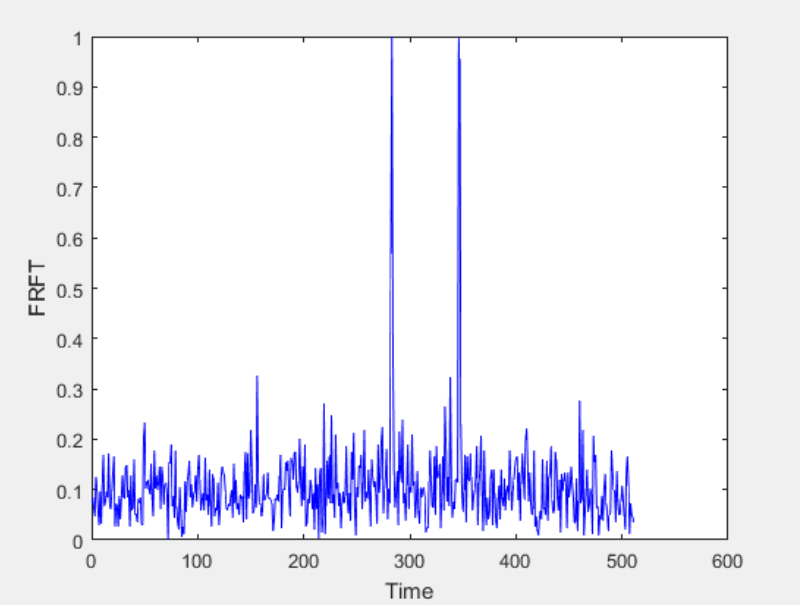

(b)

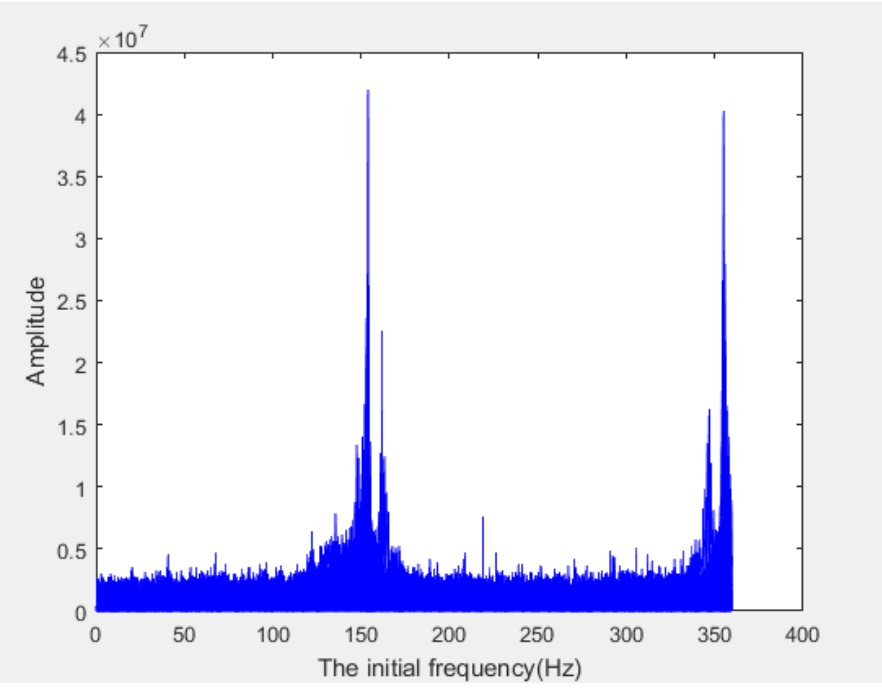

(c)

Figure 6. Spectrum distribution through the three methods under -2 dB. (a) Second-order spectrum distribution. (b) FRFT domain spectrum. (c) WHT. 
As is shown in Figure 6, that the high-order spectrum is almost unrecognizable under $-2 \mathrm{~dB}$. The recognition performance of the high-order spectrum method is bad in low SNR. The method based on FRFT is superior to high-order spectrum method, and it has good filtering performance. The recognition performance of the method based on WHT in low SNR is significantly better than the first two. Since this method is suitable for polyphase code recognition in low SNR.

\section{Conclusion}

Non-stationary signal is one of the main research objects of modern signal processing, and there are many kinds of theoretical analysis methods for it. In this paper, it is introduced the characteristics of polyphase code in time and frequency domain, and the three kinds of recognition methods of polyphase code signal, i.e. high-order spectrum, fractional Fourier transform recognition method and recognition method based on Wigner-Hough transform. The effectiveness of each method is verified by simulation, and the method based on WHT has great performance in low SNR, Which can provide reference for the identification of multi-phase code radar signals.

\section{Acknowledgements}

This work is supported partly by National Natural Science Foundation of China under Grant No. 61301205 and No. 61571146, National Defense Based Science Research Program under Grant No. JCKY2013604B001. This paper is funded by the International Exchange Program of Harbin Engineering University for Innovation-oriented Talents Cultivation.

\section{References}

[1] Yang, J., Qiu, Z.K. and Jiang, W.D. (2013) Poly-Phase Codes Optimization for Multi-Input-Multi-Output Radar. IET Signal Processing, 7, 93-100. https://doi.org/10.1049/iet-spr.2012.0195

[2] Schroer, R. (2003) Electronic Warfare. IEEE Aerospace and Electronic Systems Magazine, 18, 49-54. https://doi.org/10.1109/MAES.2003.1226535

[3] Jin, X.M. and Xu, D.Z. (2008) On Detection of Radar Polyphase Coded Waveform Based on Wigner-Hough Transform. Journal of Hefei University of Technology, No. 12, 2049-2052.

[4] Wang, X.F., Zhang, G.Y. and Rui, G.S. (2015) Detection and Parameter Estimation of the Polyphase Code Signal in a Low SNR. Journal of Xidian University, No. 1, 174-179.

[5] Du, Q. and Wang, J. (2013) Research of Detection and Parameter Estimation for P3/P4 Polyphase Codes Signal. Science \& Technology and Engineering, No. 19, 5673-5678.

[6] Jennison, B.K. (2003) Detection of Poly-Phase Pulse Compression Waveforms Using the Radon-Ambiguity Transform. IEEE Trans. on Aerospace and Electronic Systems, 39, 335-343. https://doi.org/10.1109/TAES.2003.1188915

[7] Pace, P.E. (2004) Detecting and Classifying Low Probability of Intercept Radar. Artech House, Norwood, MA, 326-329, 427-434. 
[8] Li, R., He, F.-Y. and Xia, Y.-B. (2008) Theory of Correlation Detection and Its Application. Journal of Hefei University of Technology, No. 4, 573-575.

[9] Zhang, J., Zhao, P.P. and Jia, J. (2013) Research on Relative Detection Method for Linear Frequency Modulation Signals Based on Wigner-Hough Transformation. Journal of Xihua University, No. 4, 67-70.

[10] Peacok, D.J. and Santhanam, B. (2013) Comparison off Centered Discrete Fractional Fourier Transforms for Chirp Parameter Estimation. IEEE Digital Signal Processing and Signal Processing Education Meeting, Piscataway, 65-68.

[11] Zhang, X.R. and Xie, H. (2013) A Novel Method Based on Time-Frequency Atom for Complex Modulated Radar Signal Sorting in Low SNR Condition. Applied Science and Technology, 40, 51-57.

Submit or recommend next manuscript to SCIRP and we will provide best service for you:

Accepting pre-submission inquiries through Email, Facebook, LinkedIn, Twitter, etc. A wide selection of journals (inclusive of 9 subjects, more than 200 journals)

Providing 24-hour high-quality service

User-friendly online submission system

Fair and swift peer-review system

Efficient typesetting and proofreading procedure

Display of the result of downloads and visits, as well as the number of cited articles

Maximum dissemination of your research work

Submit your manuscript at: http://papersubmission.scirp.org/

Or contactijcns@scirp.org 